PROCEEDINGS OF THE AMERICAN MATHEMATICAL SOCIETY

Volume 124, Number 11, November 1996

\title{
A NON-TREELIKE CONTINUUM THAT IS NOT THE 2-TO-1 IMAGE OF ANY CONTINUUM
}

\author{
JO W. HEATH
}

(Communicated by James E. West)

\begin{abstract}
Some thirteen years ago S. B. Nadler, Jr. and L. E. Ward, Jr., asked if any treelike continuum could be the 2-to- 1 image of a continuum. In fact, it has been conjectured that the property of being treelike characterizes those continua that are not the 2-to-1 image of any continuum. But the characterization must be something else; this paper shows that many pseudosolenoids are not the 2-to- 1 image of any continuum.
\end{abstract}

\section{INTRODUCTION}

The conjecture [8] that a continuum is the 2-to- 1 image of a continuum if and only if it is not treelike is not true. Since the Nadler-Ward question described in the abstract was raised in 1983, it has been shown that many types of treelike continua are not 2-to-1 images of continua and no one has found one that is. See [8] for a description of results on this half of the conjecture. However, we will show in Section 1 that pseudo-solenoids with infinitely many bonding maps of even degree cannot be the 2-to-1 image of any continuum. This contrasts with a construction in [7] of a 2-to-1 covering map onto the planar pseudo-circle, an example of a pseudosolenoid whose bonding maps do not have even degree. In [4] W. Dȩbski proved, using strongly the group structure of the solenoid, that there is no 2-to-1 map defined on a solenoid if infinitely many of its bonding maps are even. Although Dębski's result sounds similar, he was working with 2-to-1 domains; in fact, in [5] it was shown that every solenoid is a 2 -to- 1 retract of a continuum. In Section 2 there are theorems concerning when non-treelike continua are 2-to- 1 retracts of continua (in a nutshell: almost always if the continuum is not hereditarily indecomposable). The known results at this point leave open the following questions:

Question 1. Does there exist a non-treelike continuum that is not hereditarily indecomposable and is not a 2-to-1 retract of any continuum?

Question 2. (The big question.) Exactly which continua are 2-to-1 images of continua?

By continuum we mean a connected compact metric space. Other definitions are in a glossary just before the bibliography.

Received by the editors May 25, 1995.

1991 Mathematics Subject Classification. Primary 54C10.

Key words and phrases. Pseudo-circle, pseudo-solenoid, 2-to-1 map, treelike continuum, indecomposable continuum, hereditarily indecomposable continuum.

(C)1996 American Mathematical Society 


\section{AN EXAMPLE OF A NON-TREELIKE CONTINUUM THAT IS NOT THE 2-TO-1 IMAGE OF ANY CONTINUUM}

The construction of pseudo-solenoids was first described by J. T. Rogers, Jr. in his dissertation [11]. In that document he called all hereditarily indecomposable continua that are circularly chainable, but not chainable, "pseudo-circles". But now these continua are called "pseudo-solenoids" (even by Rogers). Rogers provided a systematic construction consisting of an inverse limit system on circles with essential, individually simplicial bonding maps. Pseudo-solenoids result if the maps are complicated enough, and he showed that all pseudo-solenoids have this structure. The definition of degree used in Theorem 1 can also be found in this paper, [11], although very little of the complexity of the definition is needed here.

Although I am sure that every English schoolgirl knows that pseudo-solenoids are not treelike, I was not able to find this fact in the literature; so Lemma 1 provides a proof. Note that the proof of Lemma 2 establishes the slightly stronger fact that the map $g$ in question is a crisp map (see definition in the glossary); we use 2-fold covering map because that is all that is needed in the proof of Theorem 1 and because covering maps are better known than crisp maps.

Lemma 1. No pseudo-solenoid is treelike.

Proof. We will use Eilenberg's theorem (see 12.38 in [9]) that says that any continuous map $f$ from a compact metric space $Y$ into $S^{1}$ is inessential if and only if there is a map $g$ from $Y$ into the reals $\mathcal{R}$ such that $f=\exp \circ g$, where exp is the map defined by $\exp (t)=(\cos (t), \sin (t))$, for each real number $t$.

Let $Y$ be a pseudo-solenoid; then by [11] $Y=\left\{\overleftarrow{Y_{i}, f_{i}}\right\}$, where each $Y_{i}$ is the unit circle. We will show that the first projection, $\pi$, that sends each point of $Y$ to its first coordinate in $Y_{1}$, is essential. Since $Y$ is one-dimensional, it will follow from the Case-Chamberlin characterization of treelike continua [2] that $Y$ is not tree like.

Suppose that $K$ is a subset of the $j$ th factor space $Y_{j}$. For this proof and the proof of Theorem 1 , we will use the $\breve{K}$ notation, $K$ enlarged, as follows:

$$
\breve{K}=\left(\prod_{i=1}^{j-1} Y_{i} \times K \times \prod_{i=j+1}^{\infty} Y_{i}\right) \cap Y .
$$

That is, $\breve{K}$ is the set of points in $Y$ whose $j$ th coordinate lies in $K$.

From the Eilenberg theorem, if $\pi$ is not essential, then there is a map $g$ from $Y$ into the reals, $\mathcal{R}$, such that $\pi=\exp \circ g$. There is a chain of open intervals $\mathcal{U}=\left\{U_{1}, U_{2}, \ldots, U_{k}\right\}$ covering the image $g(Y)$ in $\mathcal{R}$ whose links are small enough that the exp map is one-to-one on each $U_{i}$. Then there is an integer $m$ large enough that if $z$ is a point in the $m$ th circle $Y_{m}$, then $g(\breve{z})$ is a subset of an element of $\mathcal{U}$. This means that every point of the set $\breve{z}$ maps to the same point in $\mathcal{R}$ under $g$, since each point in $\breve{z}$ has the same first coordinate.

For this integer $m$, define $f_{1, m}=f_{1} \circ f_{2} \circ \ldots \circ f_{m}$, an essential map from $Y_{m}$ to $Y_{1}$, and define the map $h$ from $Y_{m}$ to $\mathcal{R}$ by $h(z)=g(\breve{z})$. The function is well defined since, as was explained above, $g$ maps the set $\breve{z}$ to a single real number. It is straightforward to see that $h$ is continuous, and the diagram commutes: $f_{1, m}=$ $\exp \circ h$. Thus, by Eilenberg's theorem again, $f_{1, m}$ is not essential.

Lemma 2. If $g$ maps the continuum $X$ exactly 2-to-1 onto a pseudo-solenoid, then $g$ is a 2-fold covering map. 
Proof. Lemma 2 in [6] states that if $g$ is a 2-to- 1 map from the continuum $X$ onto the hereditarily indecomposable continuum $Y$, then $g$ has a crisp restriction, and hence [6] a restriction that is a 2-fold covering map on a subcontinuum $S$ of $X$. The pseudo-solenoid is hereditarily indecomposable and circularly chainable, so each proper subcontinuum is hereditarily indecomposable and chainable; hence by Bing's result [1] each proper subcontinuum of a pseudo-solenoid is a pseudo-arc. Since the restriction of $g$ is 2-to-1, it cannot map onto $Y$ (unless $S=X$ ) and so the image of the restriction is a pseudo-arc. But Theorem 3 in [6] states that there is no 2-to-1 map defined on a continuum whose image is a hereditarily indecomposable treelike continuum, and hence the image cannot be a pseudo-arc. Thus $S=X$ and the map $g$ itself is a 2-fold covering map.

Lemma 3. Suppose that $g$ is a 2-fold covering map from the compact metric space $X$ onto the compact metric space $Y$. Then there is an $\epsilon>0$ such that every $\epsilon$-chain of open sets in $Y,\left\{U_{1}, U_{2}, \ldots, U_{k}\right\}$, backs up under $g^{-1}$ to two chains $\left\{V_{1}, V_{2}, \ldots, V_{k}\right\}$ and $\left\{W_{1}, W_{2}, \ldots, W_{k}\right\}$ in $X$ whose unions are disjoint and such that $g$ maps each of $V_{i}$ and $W_{i}$ homeomorphically onto $U_{i}$ for each $i=1,2, \ldots, k$.

Proof. Since $g$ is locally one-to-one, there is a positive number $\delta$ such that if $x$ and $z$ are distinct elements of $X$ and $g(x)=g(z)$, then $d(x, z)>3 \delta$. We will use the notation $N_{\theta}(t)$ to represent the $\theta$ neighborhood about the point $t$.

For each point $y$ in $Y$, there is a positive number $\epsilon(y)$ such that if $x$ and $z$ denote the two points of $g^{-1}(y)$, then $g^{-1}\left(N_{\epsilon(y)}(y)\right)$ is the union of two disjoint open sets, $E_{x}(y)$, a subset of $N_{\delta}(x)$, and $E_{z}(y)$, a subset of $N_{\delta}(z)$, and $g$ maps each of $E_{x}(y)$ and $E_{z}(y)$ homeomorphically onto $N_{\epsilon(y)}(y)$. Since $Y$ is compact, there is a single positive number, $2 \epsilon$, that works for every $y \in Y$. Now, suppose that $\left\{U_{1}, U_{2}, \ldots, U_{k}\right\}$ is an chain of open sets in $Y$ whose links have diameter no more than $\epsilon$, and for each $i$, let $y_{i}$ be a point in $U_{i}$. Denote by $V_{1}$ the subset $E_{x}\left(y_{1}\right) \cap g^{-1}\left(U_{1}\right)$ of $X$ and denote by $W_{1}$ the subset $E_{z}\left(y_{1}\right) \cap g^{-1}\left(U_{1}\right)$ of $X$. The properties of $\epsilon$ and $\delta$ not only ensure that $V_{1}$ does not intersect $W_{1}$, they also ensure that neither of the two inverse sets $V_{1}$ or $W_{1}$ can intersect both of the next two inverse sets $E_{x}\left(y_{2}\right) \cap g^{-1}\left(U_{2}\right)$ and $E_{z}\left(y_{2}\right) \cap g^{-1}\left(U_{2}\right)$. But $V_{1}$ and $W_{1}$ each must intersect at least one of the two latter sets. Accordingly, denote by $V_{2}$ whichever of $E_{x}\left(y_{2}\right) \cap g^{-1}\left(U_{2}\right)$ and $E_{z}\left(y_{2}\right) \cap g^{-1}\left(U_{2}\right)$ intersects $V_{1}$ and denote by $W_{2}$ the other. Continue naming in this way and the two chains will be identified.

Theorem 1. Suppose that $Y$ is a pseudo-solenoid whose inverse limit representation has infinitely many bonding maps with even degree. Then there is no 2-to-1 map from any continuum onto $Y$.

Proof. Suppose on the contrary that $Y$ is a pseudo-solenoid that satisfies the hypothesis and $g$ is a continuous 2-to- 1 function from a continuum $X$ onto $Y$. By

Lemma 2, we know that $g$ is a 2 -fold covering map. From [11], $Y=\left\{Y_{i}, f_{i}\right\}$, where each $Y_{i}$ is the unit circle, each individual $f_{i}$ is a simplicial map and, by hypothesis, infinitely many of the $f_{i}$ have even degree.

For the map $g$ from $X$ to $Y$ there is an $\epsilon>0$ that satisfies the statement of Lemma 3. Then, there is a positive integer $m$ such that $f_{m}$ has even degree and there is a circular chain $\left\{U_{1}, U_{2}, \ldots, U_{k}\right\}$ of intervals covering the unit circle $Y_{m}$ such that each enlarged link, $\breve{U}_{i}$, has diameter less than $\epsilon$. (The notation $\breve{U}_{i}$ was defined in the proof of Lemma 1.) Then $\left\{\breve{U}_{1}, \breve{U}_{2}, \ldots, \breve{U}_{k}\right\}$ is a circular $\epsilon$-chain covering $Y$. 
By Lemma 3 , the $\epsilon$-chain $\left\{\breve{U}_{1}, \breve{U}_{2}, \ldots, \breve{U}_{k-1}\right\}$ (all but the last link) backs up under $g^{-1}$ to two disjoint open chains $\left\{V_{1}, V_{2}, \ldots, V_{k-1}\right\}$ and $\left\{W_{1}, W_{2}, \ldots, W_{k-1}\right\}$ such that $g$ maps each of $V_{i}$ and $W_{i}$ homeomorphically onto $\breve{U}_{i}$. Each proper subchain of the circular chain in $Y$ backs up in this way, and upon reflection one sees that the entire circular chain either backs up to two disjoint circular chains (in case $V_{k}$ intersects $V_{1}$ and $W_{k}$ intersects $W_{1}$ ) or to one long circular chain (in case $V_{k}$ intersects $W_{1}$ and $W_{k}$ intersects $V_{1}$ ). Since $X$ is connected, the latter case must hold. Notice that $g$ follows the familiar 2-fold pattern of mapping the $i$ th and $(k+i)$ th links of the circular chain in $X$ homeomorphically onto the $i$ th link of the circular chain in $Y$, for $i=1,2, \ldots, k$.

Choose a point $p$ in $Y_{m}$ that lies in $U_{1}$. We will use the definition of degree from Rogers [11]. Since $f_{m}$ is a simplicial map on the unit circle $Y_{m+1}$, there are only finitely many components of $f_{m}^{-1}(p)$ and their endpoints can be labeled $\left\{e_{1}, e_{2}, \ldots, e_{n}\right\}$ in order on $Y_{m+1}$. We will temporarily define the parity of $f_{m}$ to be the parity of the number of intervals $\left(e_{i}, e_{i+1}\right)$ such that $f_{m}$ restricted to $\left(e_{i}, e_{i+1}\right)$ maps onto $Y_{m}$, including possibly the interval $\left(e_{n}, e_{1}\right)$. Note that the number of such intervals is not the same as the degree of the map which attaches +1 to those intervals that map onto $Y_{m}$ in one direction and attaches -1 to those intervals that map onto $Y_{m}$ in the other direction. Nevertheless, the parity of the function $f_{m}$ is even since its degree is even.

There is a circular chain covering the circle $Y_{m+1}$ whose links are small enough that (1) their images under $f_{m}$ refine the circular chain $\left\{U_{1}, U_{2}, \ldots, U_{k}\right\}$ covering $Y_{m},(2)$ no link contains two points of $\mathcal{E}=\left\{e_{1}, e_{2}, \ldots, e_{n}\right\}$, and (3) every link containing each $e_{i}$ is mapped by $f_{m}$ into $U_{1}$. Because we are primarily interested in the links of this circular chain in $Y_{m+1}$ that contain points of $\mathcal{E}$, we will effectively ignore the other links by labeling the circular chain $\left\{D_{1}, \ldots, D_{2}, \ldots, \ldots, D_{n}, \ldots\right\}$, where $e_{i} \in D_{i}$ for each $i=1,2, \ldots, n$. Then the enlarged circular chain $\mathcal{D}=$ $\left\{\breve{D_{1}}, \ldots, \breve{D_{2}}, \ldots, \ldots, \breve{D_{n}}, \ldots\right\}$ is still an $\epsilon$-circular chain covering $Y$ that refines the first one, $\left\{\breve{U}_{1}, \breve{U}_{2}, \ldots, \breve{U}_{k}\right\}$. Notice, for each $i=1,2, \ldots, n$, how the enlarged sets $\breve{e_{i}}$ back up: $g^{-1}\left(\breve{e}_{i}\right)=E_{i} \cup F_{i}$, two disjoint compacta in $X$, labeled so that $E_{i} \subset V_{1}$ and $F_{i} \subset$ $W_{1}$. Furthermore, each subchain $\left\{\breve{D}_{i}, \ldots, \breve{D}_{i+1}\right\}$ of $\mathcal{D}$ backs up under $g^{-1}$ to two disjoint chains. One, $\mathcal{B}_{i}$, is contained either in $\left\{V_{1}, V_{2}, \ldots, V_{k}, W_{1}\right\}$ or $\left\{V_{1}, W_{k}, W_{k-1}\right.$, $\left.\ldots, W_{1}\right\}$, but either way its first link contains $E_{i}$ and is contained in $V_{1}$. The other, $\mathcal{C}_{i}$, is contained either in $\left\{W_{1}, W_{2}, \ldots, W_{k}, V_{1}\right\}$ or $\left\{W_{1}, V_{k}, V_{k-1}, \ldots, V_{1}\right\}$, and its first link contains $F_{i}$ and is contained in $W_{1}$. (This is also true for the subchain $\left\{\breve{D_{n}}, \ldots, \breve{D_{1}}\right\}$.) Two things can happen; if $f_{m}$ restricted to the interval $\left(e_{i}, e_{i+1}\right)$ maps onto $Y_{m}$, then the last link of $\mathcal{B}_{i}$ will contain $F_{i+1}$ and will be contained in $W_{1}$, and the last link of $\mathcal{C}_{i}$ will contain $E_{i+1}$ and will be contained in $V_{1}$. If, on the other hand, $f_{m}$ restricted to the interval $\left(e_{i}, e_{i+1}\right)$ is not onto, then there is no switch from $E$ to $F$ and back. Rather the last link of $\mathcal{B}_{i}$ will contain $E_{i+1}$ and will be contained in $V_{1}$, and the last link of $\mathcal{C}_{i}$ will contain $F_{i+1}$ and will be contained in $W_{1}$.

We will build with these pieces two circular chains, $\mathcal{G}$ and $\mathcal{H}$, in $X$ whose unions are disjoint and cover $X$. This contradiction to the connectivity of $X$ will complete the proof. The sets of chains $\left\{\mathcal{B}_{1}, \mathcal{B}_{2}, \ldots, \mathcal{B}_{n}\right\}$ and $\left\{\mathcal{C}_{1}, \mathcal{C}_{2}, \ldots, \mathcal{C}_{n}\right\}$ will be divided into two camps to form the new circular chains, starting with $\mathcal{B}_{1}$ in $\mathcal{G}$ and $\mathcal{C}_{1}$ in $\mathcal{H}$. Where $\mathcal{B}_{2}$ and $\mathcal{C}_{2}$ go depends on $f_{m}$. If $f_{m}$ restricted to the interval $\left(e_{1}, e_{2}\right)$ in $Y_{m+1}$ maps onto $Y_{m}$, then there is a switch in letters: $\mathcal{B}_{2}$ goes to $\mathcal{H}$ and $\mathcal{C}_{2}$ goes 
to $\mathcal{G}$. Otherwise, if $f_{m}$ is not onto, there is no switch: $\mathcal{B}_{2}$ goes to $\mathcal{G}$ and $\mathcal{C}_{2}$ goes to $\mathcal{H}$. Either way, the first two chains in each of $\mathcal{G}$ and $\mathcal{H}$ will link up correctly at $E_{2}$ and $F_{2}$. So the general rule is this. If $f_{m}$ restricted to the interval $\left(e_{i}, e_{i+1}\right)$ in $Y_{m+1}$ maps onto $Y_{m}$, then the chain $\mathcal{B}_{i+1}$ is concatenated onto the already-assigned chain $\mathcal{C}_{i}$ and $\mathcal{C}_{i+1}$ is concatenated onto $\mathcal{B}_{i}$; that is, there is a switch in letters. On the other hand, if $f_{m}$ is not onto, there is no switch in letters; the chain $\mathcal{B}_{i+1}$ is concatenated onto the already-assigned chain $\mathcal{B}_{i}$ and $\mathcal{C}_{i+1}$ is concatenated onto $\mathcal{C}_{i}$.

Now, because the parity of $f_{m}$ is even, there are an even number of switches from $\mathcal{B}$ to $\mathcal{C}$ and back in the constructions of each of $\mathcal{G}$ and $\mathcal{H}$. This means that $\mathcal{G}$ starts with $E_{1}$ in its first link, a link from the chain $\mathcal{B}_{1}$, and ends with $E_{1}$ in its last (equal to its first) link from the chain $\mathcal{B}_{n}$. Similarly, $\mathcal{H}$ starts with $F_{1}$ in its first link, a link from the chain $\mathcal{C}_{1}$, and ends with $F_{1}$ in its last link from $\mathcal{C}_{n}$. So the chains $\mathcal{G}$ and $\mathcal{H}$ are the disjoint circular chains needed for the contradiction.

\section{Non-treelike CONTINUA that ARE 2-TO-1 RetraCtS OF CONTINUA}

If a non-treelike continuum $Y$ is not hereditarily indecomposable, then there is probably a 2-to- 1 retraction from a continuum onto $Y$. Some known theorems and the theorems in this section will explain the "probably" . For instance, Nadler and Ward [10] showed that if a continuum $Y$ fails to be hereditarily unicoherent, then $Y$ is a 2-to-1 retract (of a continuum). So if there is a simple closed curve in $Y$ for instance, or a Warsaw circle, then $Y$ is a 2-to- 1 retract. Another example: it was shown in [5] that all solenoids are 2-to- 1 retracts of continua; and note that solenoids are hereditarily unicoherent. We show here in Theorem 2 with a simple construction that if a continuum $Y$ is a 2-to- 1 retract, then so is any continuum that contains $Y$; this "superset" phenomenon greatly expands the set of examples of continua that are known to be 2-to-1 retracts. Then we show in Theorems 3 and 4 that each hereditarily decomposable non-treelike continuum is a 2-to- 1 retract, but no hereditarily indecomposable continuum (treelike or not) is a 2-to- 1 retract. Also we demonstrate in Theorem 5 how the existence of an essential map onto the unit circle with at least one connected point inverse guarantees a 2-to-1 retraction.

Theorem 2. Suppose that the continuum $Y$ is a 2-to-1 retract of a continuum, and suppose that $Y \subset Z$. Then $Z$ is also a 2-to-1 retract of a continuum.

Proof. Let $r$ denote a 2-to-1 retraction from the continuum $X$ onto $Y$. Let $Z_{1}$ and $Z_{2}$ denote two copies of $Z$, with the corresponding copies of $Y$ named $Y_{1}$ and $Y_{2}$, and let $X_{1}$ denote a copy of $X$ with $Y_{3}$ its copy of $Y$. We may assume that $Z_{1}$, $Z_{2}$, and $X_{1}$ are disjoint. Now let $W$ denote the union of $Z_{1}, Z_{2}$, and $X_{1}$ with $Y_{1}$, $Y_{2}$, and $Y_{3}$ identified into a single copy, say $Y_{4}$, of $Y$. There is a natural 2-to-1 retraction of $W$ onto $Z_{1}$ that uses $r$ (or a copy of $r$ ) from $X_{1}$ onto $Y_{4}$ and matches $Z_{2} \backslash Y_{2}$ with $Z_{1} \backslash Y_{1}$.

Theorem 3. If $Y$ is a hereditarily decomposable non-treelike continuum, then there is a continuum that retracts exactly 2-to-1 onto $Y$.

Proof. This follows immediately from H. Cook's theorem [3] that all $\lambda$-dendroids are treelike. A $\lambda$-dendroid is a hereditarily decomposable and hereditarily unicoherent continuum. So if $Y$ is a hereditarily decomposable non-treelike continuum, then it cannot be hereditarily unicoherent, and the conclusion of Theorem 3 follows from the Nadler-Ward result described in this section's opening paragraph. 
Theorem 4. No hereditarily indecomposable continuum is a 2-to-1 retract of a continuum.

Proof. Suppose that the hereditarily indecomposable continuum $Y$ is a 2 -to- 1 retract of a continuum $X$. Let $r$ denote the retraction. As was used earlier in this paper, any 2-to-1 map from a continuum onto a hereditarily indecomposable continuum has a crisp restriction to a continuum in the domain ([6]). And, also from [6], each crisp map is a 2- fold covering map. So the restriction is a 2 -fold covering map from a subcontinuum $A$ of $X$ onto a subcontinuum $B$ of both $A$ and $Y$. Now, the connected set $A$ is equal to $(A \backslash B) \cup B$, two disjoint sets with the second set closed. Hence there is a point $p$ in $B$ that is a limit point of $A \backslash B$. Let $\left\{p_{i}\right\}$ denote a sequence of points in $A \backslash B$ that converges to $p$. By the continuity of $r$, the sequence $\left\{r\left(p_{i}\right)\right\}$ converges to $r(p)$ which is $p$ since $r$ is a retraction. Note that $r\left(p_{i}\right) \neq p_{i}$ since the former is in $B$ and the latter is in $A \backslash B$; hence there are, arbitrarily close to $p$, two points of $A$ that map the same under $r$. This means that the restriction of $r$ is not locally one-to-one and so cannot be a 2 -fold covering map. This contradiction completes the proof.

Theorem 5. Suppose $g$ is an essential map from the continuum $Y$ onto the unit circle $S^{1}$ such that for some point $p$ in $S^{1}$, the inverse $g^{-1}(p)$ is connected. Then $Y$ is a 2-to-1 retract of a continuum.

Proof. Since the points of the unit circle $S^{1}$ are determined by their polar angle, we will simplify the notation by assuming that $g$ maps $Y$ onto $S^{1}=(0,2 \pi]$, and we'll try to remember that $2 \pi$ is a limit point at the 0 end.

Suppose now that $g^{-1}(2 \pi)=M$ is a continuum in $Y$. Construct the space $Z \subset Y \times[0,2 \pi]$ by

$$
Z=(Y \times\{0\}) \cup\{(y, g(y)) \mid y \in Y\} .
$$

We will think of $Y \times\{0\}$ as the original space $Y$. The map that sends both $(y, 0)$ and $(y, g(y))$ to $(y, 0)$ is a 2-to- 1 retraction from $Z$ onto $Y \times\{0\}$. But, is $Z$ a continuum? The continuity of $g$ ensures that $Z$ is compact. Suppose that $Z=A \cup B$, two disjoint open and closed sets. One of them, say $A$, contains the connected set $Y \times\{0\}$. If $B$ does not intersect $M \times\{2 \pi\}$, then there are angles $\alpha$ and $\beta$ with $0<\alpha<\beta<2 \pi$ such that all of the second coordinates of points of $B$ lie in the interval $[\alpha, \beta]$. Define the natural projection $\pi$ from $Y \times(0,2 \pi]$ down to $Y \times\{0\}$ by the obvious formula $\pi(y, \theta)=(y, 0)$. Then $\pi(B)$ is both open and closed in $Y \times\{0\}$ but does not contain $Y \times\{0\}$. This contradicts the fact that $Y$ is connected. Thus the connected set $M \times\{2 \pi\}$ intersects $B$ and thus is a subset of $B$. Since $B$ is separated from $M \times\{0\}$, there is an angle $\alpha>0$ such that the second coordinate of any point of $B$ is greater than $\alpha$, and similarly, since $A$ is separated from $M \times\{2 \pi\}$, there is an angle $\gamma<2 \pi$ such that the second coordinate of any point of $A$ is less than $\gamma$.

We will show that this structure makes the map $g$ inessential; a contradiction that implies that $Z$ must be connected. The space $Y \times\{0\}$ is the union of two closed sets, $B_{1}=\pi(B)$ and $A_{1}=\pi(A \cap(Y \times(0,2 \pi])) \cup(M \times\{0\})$, whose intersection is $M \times\{0\}$. We define two homotopies, $H_{1}$ on $B_{1} \times[0,1]$, and $H_{2}$ on $A_{1} \times[0,1]$, both into $S^{1}$, such that these two homotopies agree (in fact are constant) on the intersection, $M \times\{0\}$, of their domains and the two homotopies end with the same constant map. Their union is a homotopy from $g$ to a constant map. For each 
$t \in[0,1]$, define:

$H_{1}(b, t)=2 \pi t+(1-t) g(b)$ for $b \in B_{1} \backslash(M \times\{0\})$ and $H_{1}(m, t)=2 \pi$ for $m \in M \times\{0\}$

and

$H_{2}(a, t)=(1-t) g(a)$ for $a \in A_{1} \backslash(M \times\{0\})$ and $H_{2}(m, t)=0$ for $m \in M \times\{0\}$.

\section{Definitions}

1. Chain (Circular chain). A chain (circular chain) of sets, called links, is a finite collection that can be indexed $\left\{S_{1}, S_{2}, \ldots, S_{k}\right\}$ so that $S_{i}$ intersects $S_{j}$ if and only if $|i-j| \leq 1$ (except that for circular chains $S_{1}$ intersects $S_{k}$ ).

2. Chainable (Circularly chainable). A continuum is chainable (circularly chainable) if for each $\epsilon>0$ there is an $\epsilon$-chain ( $\epsilon$-circular chain) of open sets covering the continuum.

3. Continuum. A topological space is a continuum if it is connected, compact, and metric.

4. Crisp. A map $f$ is crisp if, for each proper subcontinuum $C$ in the image, there are exactly two components of the preimage of $C$ and $f$ maps each of these components homeomorphically onto $C$.

5. Degree of a map. For the definition of the degree of a simplicial map from $S^{1}$ onto itself, see [11].

6. Essential map. A map is essential if it is not homotopic to a constant map.

7. $\epsilon$-chain. A chain is an $\epsilon$-chain if each link has diameter less than $\epsilon$. And the same holds for $\epsilon$-circular chain.

8. $\epsilon$-map. An $\epsilon$-map is a continuous function whose point inverses have diameter less than $\epsilon$.

9. Pseudo-solenoid. A continuum is a pseudo-solenoid if it is hereditarily indecomposable and circularly chainable but not chainable.

10. Solenoid. A continuum that is an inverse limit of circles such that each bonding map is an $n$-fold covering map for some integer $n$. A solenoid that is not a circle is indecomposable and each proper nondegenerate subcontinuum of any solenoid is an arc.

11. 2-to-1. A function is 2-to-1 if the preimage of each point in the image has exactly two points.

12. Treelike. A continuum is treelike is for each $\epsilon>0$, there is an $\epsilon$-map from the continuum onto a tree (an acyclic graph).

\section{REFERENCES}

[1] R. H. Bing, Concerning hereditarily indecomposable continua. Pacific J. Math. 1 (1951) 43-51. MR 13:265b

[2] J. H. Case and R. E. Chamberlin, Characterizations of tree-like continua. Pacific J. Math. 10 (1960) 73-84. MR 22:1868

[3] H. Cook, Tree-likeness of dendroids and $\lambda$-dendroids. Fund. Math. 68 (1970) 19-22. MR 41:6171

[4] W. Dębski, Two-to-one maps on solenoids and Knaster continua, Fund. Math. 141 (1992) 277-285. MR 94b:54094

[5] W. Dębski, Jo Heath, J. Mioduszewski, Exactly 2-to-1 maps onto arc continua, To appear, Fund. Math.

[6] Jo Heath, 2-to-1 maps with hereditarily indecomposable images. Proceedings of the Amer. Math. Soc. 113 (1991)839-846. MR 92c:54012 
[7] Jo Heath, Weakly confluent, 2-to-1 maps on hereditarily indecomposable continua. Proceedings AMS 117 (1993) 569-573. MR 93d:54047

[8] Jo Heath, Exactly k-to-1 maps: from pathological functions with finitely many discontinuities to well-behaved covering maps. Continua with the Houston Problem Book, Lecture Notes in Pure and Applied Mathematics, Series/170, Marcel Dekker, New York, 1995, pp. 89-102. MR 96d:54015

[9] Sam B. Nadler, Jr. Continuum Theory. Marcel Dekker, Inc. New York, Basel, Hong Kong. MR 93m:54002

[10] S. B. Nadler, Jr. and L. E. Ward, Jr., Concerning exactly (n,1) images of continua, Proceedings AMS 87 (1983), 351-354. MR 84c:54059

[11] J. T. Rogers, Jr. Pseudo-circles and universal circularly chainable continua. Ill. J. Math. 14 (1970) 222-237. MR 41:9213

Department of Mathematics, Auburn University, Auburn, Alabama 36849-5310

E-mail address: heathjw@mail.auburn.edu 